\title{
Analysis and optimisation of railway nodes using simulation techniques
}

\author{
A. Kavička ${ }^{1}$, V. Klima ${ }^{2} \&$ N. Adamko ${ }^{2}$ \\ ${ }^{1}$ Jan Perner Transport Faculty, University of Pardubice, \\ Czech Republic \\ ${ }^{2}$ Faculty of Management Science and Informatics, University of Žilina, \\ Slovak Republic
}

\begin{abstract}
Railway nodes represent sensitive points of railway networks, the efficiency of which influences the effectiveness of network processes. Those nodes belong to the most complex service systems involving sophisticated technological processes and are equipped with quite complicated and costly technical devices. Design of those systems and the organization of their operation have to facilitate the required capacity together with a high quality of service processes as well as minimal costs of resources. That intention can be achieved using various measures, e.g. track infrastructure modification, utilization of alternative kinds of resources, improvement of scheduling and rostering, enhancement of technological processes, adjustment of decision making strategies or entire system reengineering (that approach can be applied to a design of a new node). The application of exact mathematical optimisation methods is quite limited because of the high complexity of the above-mentioned systems. Computer simulation offers a quite flexible and credible technique eliminating the disadvantages of precedent approaches, i.e. a simulation model of railway node (substituting an existing or planned system) enables one to investigate all mentioned ways of an increase of node capacity. Efficient methodologies are introduced aimed at the solution of capacity problems related to railway nodes using the simulation software tool Villon, which was originally determined for simulations of railway marshalling yards; however its flexible hierarchical software architecture enabled us to extend its scope to other kinds of transportation nodes (including e.g. automobile traffic).
\end{abstract}

Keywords: transportation node, simulation model, planning, optimisation. 


\section{Transportation terminals}

The most important and evidently the most costly part of a logistic chain is the transfer process. This part is often called transport logistics. The transfer process itself is composed of a movement along the transportation route and of necessary manipulations (service processes) applied to transportation means and to transported commodities at specialised locations called (transport) terminals. At the terminals, individual entities may originate, disappear, and be subject to service or to transformation. Several kinds of elements are distinguished in the terminal structure:

- Terminal infrastructure (transportation paths, security and information elements, buildings and other static equipment, e.g. platforms, parking lots, etc.),

- Mobile service resources (human resources, transport and manipulation devices, etc.),

- Elements subject to a service (cars, railway carriages, containers, passengers, etc.) and

- Control system for service processes.

Undoubtedly, the most complicated situation from the point of view of manipulation and process control occurs in terminals, where the infrastructure is composed mostly of set of tracks, where the mobile service resources are mostly locomotives and where the elements under service are also mostly railway vehicles. Here belong railway logistic terminals and terminals with a significant portion of a railway infrastructure. Exactly the terminals with these characteristics are in the focus of our attention.

Let us see examples of the railway logistic terminals, where the common problems concerning the infrastructure design and planning and the traffic control appear, and where also the similar approach to their solution is applicable:

- $\quad$ Marshalling yards contain usually only the trackage and the prevalent activities are transformation operations with the rail vehicles (coupling, uncoupling, sorting, various kinds of shunting, etc.).

- Passenger railway stations are also equipped with trackage, but the prevalent activities are the service operations (entering and leaving the carriages, cleaning, etc.). The elements subject to a service are passenger carriages and also passengers in the entrance hall.

- Industrial sidings are often combined with road transport and so they contain infrastructure, service resources and elements subject to service of both types. In heavy industry plants, the set of tracks may be extremely large and may even contain an independent train forming part. Here, complex operations are characteristic, namely complicated manipulations and also loading and unloading of different substances.

- Specialized railway logistic centres serve usually for maintenance, reparations, cleaning and other service and for completion of train sets (railway depots). 
- $\quad$ Terminals for multimodal transport (e.g. container terminals) contain also a heterogeneous infrastructure and various elements (railway, road, water transport elements).

- Transport nodes, which, from the point of view of infrastructure problems and traffic operation problems, should be understood in a broader sense as an integration of several types of terminals. For example, a need may arise to integrate goods railway station, passenger railway station and a corresponding depot into a single transport node.

\section{Problems of infrastructure planning}

Generally, regardless the terminal type, the problem of infrastructure planning (e.g. trackage design) inside the terminal presents the task to define elements of the infrastructure and the task to integrate them into a system. The infrastructure planning need arises either in the realisation of long-term strategic aims or in the optimisation of the operation of standing terminals.

Among strategic aims are namely:

- Decision to construct a new terminal or a new part of a terminal.

- Technical reconstruction of a terminal infrastructure, usually followed by modernisation of its elements.

- Enlargement of infrastructure with the aim to increase its capacity, if the service increase is expected.

- Decision to reduce the active infrastructure if the service depression is expected.

- Decision to concentrate investments into modern and well equipped large terminals and to suppress operations in other terminals.

Among operational problems in infrastructure planning are:

- Terminal operation is too costly or terminal is working below required capacity while solutions via optimisation of mobile resources and via optimisation of technological processes are exhausted.

- $\quad$ It is evident that the infrastructure is not correctly balanced, i.e. some parts are insufficiently dimensioned and are causing operational bottlenecks while other are over dimensioned and are not sufficiently exploited.

The problem of infrastructure planning is in other words a problem of optimal configuration of the terminal infrastructure. A correct solution of an optimisation problem requires, of course, definition of an optimisation criterion. But in this case it is not easy at all and we even do not intend to try to do it. Instead we will point out some objectives we are trying to achieve, which are often contradictory:

a) Minimal infrastructure size is desirable because of significant construction, maintenance and operational costs.

b) Sufficient size of infrastructure must be defined to cope satisfactorily with a stochastic nature of required service volume. 
c) Configuration of infrastructure elements should be balanced to avoid bottlenecks on one side and idle parts on the other.

d) Infrastructure configuration should have "reasonable" reserves of capacity to cover long term or short term increase of service requirements and random jumps.

\section{Problem of optimal infrastructure configuration}

Any solution of optimisation of infrastructure configuration should observe the following facts:

- Realisation of an accepted solution is typically extremely costly and thus the solution should be a long term one. It is practically impossible to make additional corrections in the case of a wrong decision.

- Infrastructure is only a resource, which serves the goal - traffic operation. Configuration of an infrastructure is satisfactory only if the operation on it is effective. Therefore, if we want to judge the suitability of an infrastructure, we have to know and to describe in detail (make a model) the traffic realized on it.

- The traffic operation of a terminal is an extremely complex system. It is a dynamic system with complex relations among its elements where the service processes are mutually intertwined mostly with stochastic interactions.

The question is what can be done from the management side to ensure the most objective decision and to avoid an erroneous decision.

Due to the complexity of the system and its stochastic behaviour, the use of exact mathematical methods is significantly limited. Classic planning methods, on the other side, use simplified models of traffic operation which do not take into account the stochastic features of the system and do not involve detailed behaviour of mutually dependent dynamical technological processes. Instead, mean and normative values are used together with expert knowledge based on past experience. Frequently, the result is a solution which, confronted with a real traffic, appears to be unsuitable.

Where can we find a way out of this situation? Exact mathematical solution, as we can see, is not practicable. The only feasible way is to accept a good suboptimal solution and to find it in an environment, which is capable to reflect satisfactorily the complexness of the infrastructure and of the traffic and at the same time it is capable to produce understandable solutions based on verified arguments. Experimental environment which satisfies these conditions is a simulation model of a terminal, embodied in hardware and software form as a substitute for a real or designed terminal, which respects exactly the infrastructure composition and describes in detail all traffic processes in their complexity. Simulation model is used as an experimental environment with the objective to evaluate all consequences of proposed infrastructure configuration. Evidently, the effectiveness of the traffic is a criterion of the evaluation. 


\section{Simulation techniques}

Simulation of systems is a method supporting analysis, design and optimisation of real systems. Three steps can be distinguished:

1. Substitution of a real system by its simulation model.

2. Experimenting with the simulation model with the aim to disclose its properties, behaviour and reactions under selected conditions.

3. Application of obtained results in the real system (existing or designed)

Simulation model should be as accurate and detailed as possible so that its results are applicable to the reality. At the other side, there is also certain limit of detail, which should not be trespassed.

The software product Villon belongs to simulation tools which are based on above described principles and was successfully verified in practice. Villon is not only a very efficient tool for verification of infrastructure modifications but it is also very suitable mean for investigation of possibilities how to increase the efficiency of service resources and improve decision activities. Villon enables the user to construct complex, detailed and interactive simulation models, make experiments with them and analyse the results. Simulation model developed in the frame of experimental environment of the tool Villon can be an effective aid for solution of above described problems of infrastructure planning, both strategic and operational.

The tool Villon itself, however, does not give automatically ready-to-use optimal solutions of complex problems but offers an experimental environment, a "laboratory" for investigation of various configurations of a terminal infrastructure configuration and all consequences of their application. Simply said, using Villon, a user-experimenter can answer questions "What happens, if ...?". It is supposed that the user himself is expert in technology and, moreover, cooperates closely with the management officers of the terminal.

Several basic stages of work with Villon can be distinguished:

a) Acquisition, processing and analysis of data concerning real or planned terminal.

b) Construction of infrastructure model (object of investigation).

c) Construction of dynamical operation (traffic) model, which serves for investigation of the terminal properties with proposed infrastructure configuration.

d) Experimenting with the simulation model. It is an iterative process of consequent runs of simulation experiments with modified parameters with the objective to disclose the behaviour of the system in correspondence with the given set of parameters leading to the solution of the problem. It is evident that a number of simulation experiments should be performed. This number depends on the degree of expertise of experimenter, on the quality of the simulation model and also on tools available for evaluation of experiments. A correct evaluation of results undoubtedly shows the direction and next steps in the quest for solution.

e) Analysis of results of simulation experiments. Villon enables the user to follow on-line animation of movements of all mobile resources and of 
technological activities. Moreover, Villon is capable to submit information on properties of all both static and dynamic terminal elements including clients of the system, via so called Explorer. The broad scope of tools includes the possibility to obtain up-to-date statistics about the performance (state) of all mobile and fixed elements of the terminal. This information is given in a graphic form.

It is clear that during the simulation run we are not able to discover all aspects of the system behaviour. Villon however offers the possibility of recording simulation run into a simulation protocol which can be used later as a data source for post-simulation evaluation of results. Again, Villon offers a user a set of tools for statistical evaluation of protocol data, for construction of schedules of resources activities in graphic form, exploitation of infrastructure, etc. If needed, all recorded data can be exported e.g. to MS Excel format.

Credibility of the experiment results strongly depends on the good fit of the infrastructure model and of the traffic operation model to the reality. In the following two sections these models and their properties are dealt with more closely.

\section{Model of infrastructure}

Steps leading to the construction of an infrastructure model are best explained on the example of building the model with the most complicated infrastructure, which is a railway (track) infrastructure. The infrastructure model (fig. 1) is created by transformation of a map (plan) which is available either on paper or in electronic form. The obtained infrastructure model guarantees an absolute accuracy of the fit. Thanks to it, an accurate, not only simplified model of trackage is used in the simulation and therefore we can evade distortions caused by rough approximations of distances and their influence on movements of mobile elements in the simulation model.

Transformation of original plans constitute so called physical level of an infrastructure model. It means that a trackage composed of basic elements, tracks, simple switches, cross switches, track crossings, is available to the model builder (tracks can be divided into sections corresponding to the trackage organisation or into isolated sections for the signalling system). In this level, however, professions of individual tracks are not yet defined. The notion "profession" means the purpose for which the tracks serve in the trackage, e.g. arrival of terminating trains, connecting tracks to depots for uncoupled train locomotives, hump tracks, sorting tracks, etc. Information on the track profession is important for the model building in the phase of technology definition and definition of technological activities of the service of train sets. For example, in the technological activity "Transfer of shunting locomotive to the train set", profession of track, used for this transfer, must be defined. Professions are assigned to individual track in accordance with the track professions in the trackage of the real infrastructure. Assignments of professions constitute a logical level of the infrastructure model. 


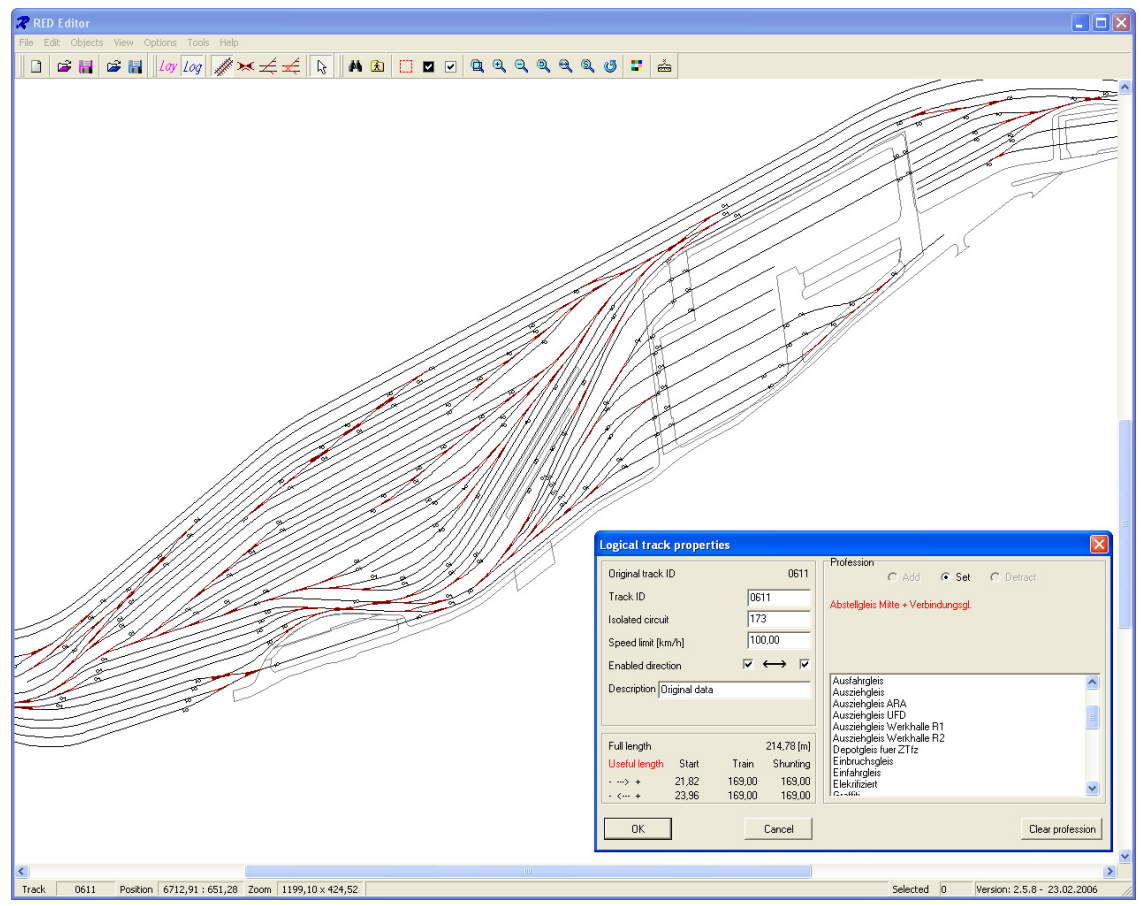

Figure 1: User interface of the programme RED presenting a detail depot infrastructure and a track properties dialog box.

After physical level of the infrastructure model, list of track professions and logical level of the infrastructure are defined, routes for the mobile elements are created. Moreover, also dynamically ad hoc created routes may be used, which are found automatically during the simulation experiment, where an actual availability of tracks is considered.

\section{Traffic operation model}

To verify the suitability of a proposed configuration of infrastructure in a real terminal, its operational verification is important. It is done by a series of simulation experiments with various sets of traffic conditions. These simulation experiments may reveal eventual flaws in the infrastructure configuration, which may cause, that expected performance of the terminal or the smooth run of local technological processes are not reached. Then, another series of experiments is performed with modified configuration aimed at elimination of ineffectiveness. In an extreme case even the misconception of the whole infrastructure can be proved by the simulation experiments and its reconstruction is necessary. Of course, this approach is very valuable for eventual investors, who will decide about their investments in the infrastructure construction on the basis of successful simulation verification. Once more we stress that a good fit to reality 
must exist, all important factors concerning real traffic operation must be observed. In the simulation tool Villon, dynamic model of the terminal traffic operation consists of the following components:

a) Mobile service resources, which include individual workers, operating in the terminal field (e.g. shunters, couplers, external transiteurs, locomotive guards, etc.), locomotives (e.g. shunting, hump or train locomotives, etc.).

b) Technological processes formally describing standard technological processes, including requirements for assignment of necessary resources. Good examples of a suitable and clear formal presentation of technological processes are so called networks (directed acyclic graphs).

c) Objects under service, describing temporary elements subject to various type of service is given (e.g. trains or wagons, trucks, containers, etc.).

d) Operations control, focused namely to (i) setting priorities in processing of individual objects under service, (ii) management of all types of service resources, i.e. of infrastructure and of mobile elements which are assigned to or taken away from clients - service processes, with respect to given rules.

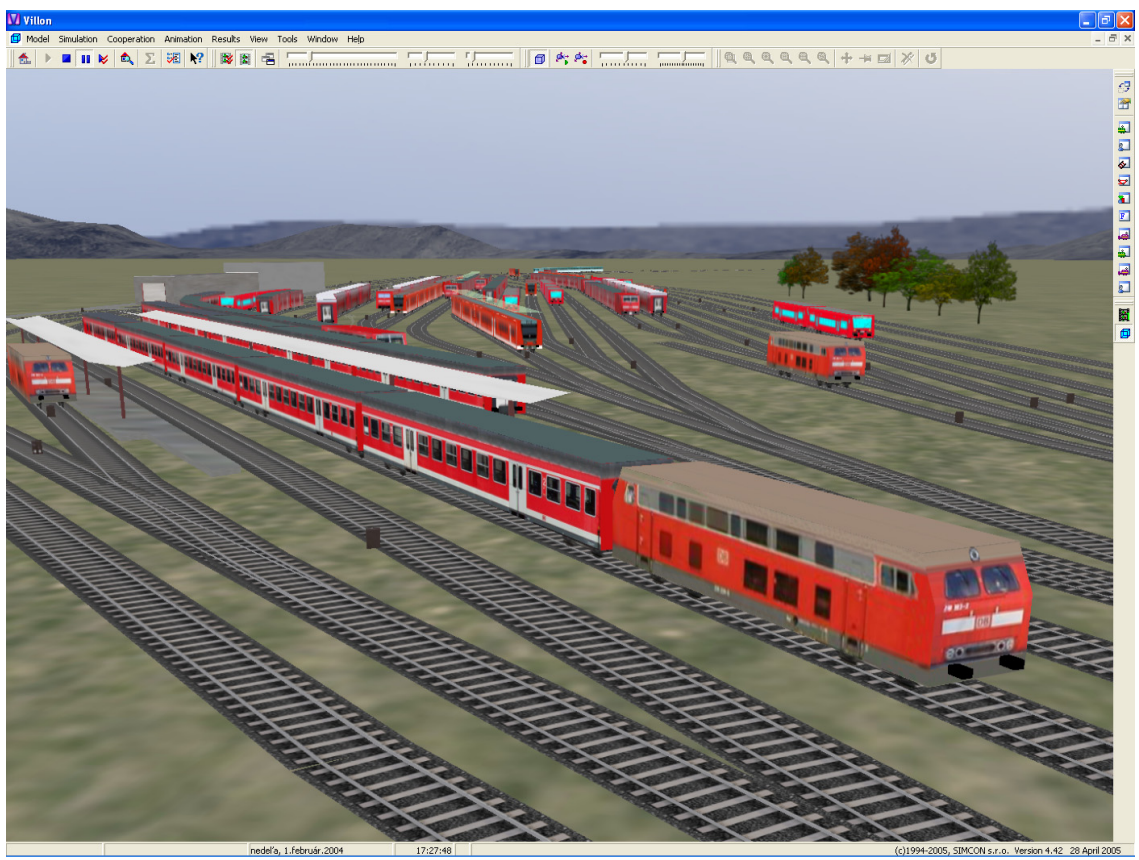

Figure 2: Animation of vehicle movements.

Experimental simulation environment of the tool Villon illustrates the operation of investigated traffic using an on-line animation (fig. 2), which enables a detailed following of its development during the working period and offers a broad scope of post simulation statistical evaluations of the experiment results. 


\section{Applications}

In section 2, typical problems in the infrastructure planning were presented, where simulation technique may be an efficient tool for their solution. Simulation tool Villon assisted successfully to the solution of most of these problems, always with a significant economic profit.

Villon was used for example for the infrastructure planning and verification of traffic operation in the train set depot in Ulm, Germany. Here, the problem of trackage configuration was complicated by problems of location and configuration of individual service modules of the depot (internal and external cleaning station of train sets, fuel supply device, repair and maintenance facilities, etc.). Another example of the Villon successful application in planning a new terminal is Slovak marshalling yard Teplička nad Váhom near Žilina, whose importance at present is increasing substantially.

Changes in the organisation of train forming system in Austrian and Swiss railways led to cancelling of several small marshalling yards and modernisation and extension of greater and more efficient ones. The customer flows originally processed in the cancelled stations had to be redirected into the remaining yards. Villon was used for verification of suitability and efficiency of new infrastructure solutions in Linz (Austria) [9], Basel and Lausanne (Switzerland).

Attention was also paid to infrastructural and technological changes connected with the traffic operation of passenger railway stations [2]. Last year, Villon was applied with success in the investigation of planned traffic operations in Prague, Masaryk railway station, in Brno (design of a new railway station), both in Czech Republic, and in one passenger railway station belonging to Beijing railway node in the People's Republic of China.

Industrial railways must be flexible enough to be capable to cope with an increase or decrease of company production, which immediately reflects on the intensity of transport activities. However, modification of infrastructure is often limited by geographical conditions or by financial reasons. The tool Villon was successful even in this area and was applied in the solution of problems following the production increase in paper production plant SCA Laakirchen, Austria, steelworks Corus Teesside in the UK and in infrastructure modification process in giant chemical plant BASF Ludwigshafen, Germany.

For a complex and detailed investigation of infrastructure of some terminals, an interaction between railway transport and other transport modes (e.g. road transport) should be considered. Villon was utilized to verify different configurations of road and railway infrastructure in Volkswagen Bratislava plant. The entire internal transport was modelled, including trucks, cars, trains and pedestrians. Austrian steel works VOEST Alpine Linz needed a simulation study of a road infrastructure reconstruction in interaction with the railway and road traffic inside the plant area.

Once built, verified and validated model of a terminal can be used repeatedly in seeking for solutions of a different type [1,3]. For example, simulation model which was originally developed as a tool for optimal reconstruction of a terminal (strategic goal) can be also useful during the real 
reconstruction process in the solution of problems caused by planned closings due to reconstruction.

\section{Summary}

Planning and optimisation of infrastructure and of traffic processes inside the terminals and transport nodes is unthinkable without detailed and objective evaluation of consequences which follow every decision, because extremely high money funds are at stake. The suitability of planned changes of infrastructure respectively of technological procedures cannot be evaluated objectively without a thorough study of the terminal operation after the changes are realised. Perhaps, due to extreme complexity of the terminals and their operation, the only efficient technique how to evaluate and verify all consequences of our decisions is making experiments with well fitted simulation model of the terminal.

\section{Acknowledgements}

This work has been supported by the Czech research project MSM 0021627505 "Theory of transportation systems" and by the Slovak grant project 1/1049/04 "Agent-based architecture of simulation models related to service systems".

\section{References}

[1] Kavička, A., Klima, V.: ABAsim: Agent-based architecture of simulation models, Simulation Almanac 2005, Czech and Slovak Simulation Society, Prague, pp.63-72, 2005

[2] Bažant, M., Kavička, A.: Simulation model of Prague main station, Proc. of international symposium “ŽEL 2005”, University of Žilina, pp.18-22, 2005

[3] Kavička, A., Klima, V., Adamko, N.: Agent-based simulation of transportation nodes, EDIS, University of Žilina, 2005, (in Slovak),

[4] Adamko, N., Klima, V., Márton, P., Kavička, A.: Simulation Support of Railway Station Infrastructure Design, Transport - Prace Naukowe, Wydawnictwo Institutu Technologii Eksploatacji, Radom, pp. 11-16, 2005

[5] Márton, P., Klima, V., Kavička, A., Adamko, N.: Simulation support of railway infrastructure in logistics terminals planning, Proc. of international symposium Transport 2004, Univ. of Transport, Sofia, pp. 489-494, 2004

[6] Xu, Z.L., Klima, V., Kavička, A.: The Realization of Joint Terminal Process Simulation by Computer, Railway Transport and Economy, China Academy of Railway Sciences, 1/2003, Beijing, pp.51-53, 2003 (in Chinese)

[7] Kavička, A., Klima, V.: Simulations of railway station operation, Proc. of the World congress on Railway Research, Köln, 2001

[8] Klima, V., Kavička, A.: Simulation support for railway infrastructure design and planning processes, Proc. of Comprail 2000 conference, Wessex Institute of Technology, Southampton-UK, pp.447-456, 2000

[9] Kavička, A., Klima V., Niederkofler, A., Zat'ko, M.: Simulation model of marshalling yard Linz Vbf (Austria), Proc. Int. Workshop on Harbour, Maritime \& Logistics Modelling and Simulation, SCS, Genoa, pp.317-320, 1999 